\title{
Eccentric-fluted beam pipes to damp quadrupole higher-order modes
}

\author{
M. Sawamura \\ Japan Atomic Energy Agency, Tokai, Ibaraki 319-1195, Japan \\ T. Furuya, H. Sakai, T. Takahashi, and K. Umemori \\ High Energy Accelerator Research Organization (KEK), Tsukuba, Ibaraki 305-0801, Japan \\ K. Shinoe \\ ISSP, University of Tokyo, Kashiwa, Chiba 277-8581, Japan
}

(Received 3 November 2009; published 23 February 2010)

\begin{abstract}
An eccentric-fluted beam pipe is proposed to damp quadrupole modes in a cavity. The eccentric flutes act as a mode converter from quadrupole to dipole. Optimizing the parameters of the eccentric-fluted beam pipe allows sufficient damping of both degenerate quadrupole modes. The external $Q$ values of the eccentric-fluted beam pipe measured with a low power model cavity agree well with those calculated with the 3D electromagnetic field simulation code MAFIA.
\end{abstract}

DOI: 10.1103/PhysRevSTAB.13.022003

PACS numbers: 29.20.Ej

\section{INTRODUCTION}

The superconducting rf accelerator is one of the fundamental technologies for present and future accelerator facilities such as the International Linear Collider, European X-ray Free-Electron Laser, Energy-Recovery Linacs (ERLs), and the Spallation Neutron Source [1]. The intrinsic advantage of the superconducting rf accelerator is its extremely high $Q$ values (low loss) for the cavity eigenmodes. Owing to the high $Q$ values, the superconducting accelerator can generate high-power charged particle beams with negligibly small heat losses dissipating in the cavities. The high $Q$ values of superconducting cavities, however, cause a troublesome problem, resonant excitation of higher-order modes (HOMs) in the cavity. The amplitude of the fundamental mode, i.e., the accelerating mode, stored in the cavity is controlled to be constant by balancing the beam load and the input power fed through the main coupler. HOMs excited in the cavity, however, cannot be controlled in the same manner.

The resonant excitation of HOMs is a crucial issue especially in high-current $\mathrm{cw}$ machines such as ERLs. HOMs with high $Q$ values induce beam breakup (BBU) instabilities [2] and limit the beam current. This BBU instability was observed experimentally at the JLab FEL upgrade [2]. Increasing the beam current caused a growth of HOM voltage and beam losses. The BBU threshold current was found to be $2.7 \mathrm{~mA}$ in this measurement. The multipass BBU instabilities in a recirculation linac have been analyzed theoretically [3,4]. The threshold current of the BBU instability caused by dipole HOMs can be analytically obtained for the simplest configuration consisting of a recirculation linac, a single cavity, a single HOM, and a single loop. The threshold current is inversely proportional to the HOM $Q$ value.
In order to develop high-current superconducting accelerators of several $100 \mathrm{~mA}$ needed for such devices as the future ERL light source, suppression of the HOM BBU is one of the most important issues. In a design of a $5-\mathrm{GeV}$ ERL, a Cornell University group numerically studied HOM BBU and found criteria of shunt impedance and $Q$ values for monopole, dipole, and quadrupole HOMs to achieve a $100 \mathrm{~mA}$ beam in the main linac [5]. They pointed out that the quadrupole mode damping as well as the dipole modes damping is important.

One possible way to damp HOMs is to enlarge the beam pipes connected to both ends of the cavity. Table I shows the relation between the cutoff frequencies and the beam pipe diameter of the TESLA cavity [6], for example. The three lowest cutoff frequencies correspond to the TE11 (dipole), TM01 (monopole), and TE21 (quadrupole) modes. Enlarging the beam pipe diameter enables these HOMs to propagate along the beam pipe. The quadrupole mode requires the largest diameter among three modes as shown in Table I. Increasing the beam pipe diameter to propagate quadrupole modes causes the deeper penetration of the fundamental mode into the beam pipes. This results in longer distance from the cavity to the HOM absorber and a lower packing factor.

Fluted beam pipes are another solution to damp HOMs [7]. They also lower the cutoff frequencies of the HOMs, but are insufficient to control quadrupole HOMs.

We propose an asymmetric fluted beam pipe, called here an eccentric-fluted beam pipe (EFB), to solve these difficulties in achieving quadrupole damping. The EFB is formed by displacing the direction of the flute away from the beam pipe center and/or by folding around the midpoint of the flute to couple with quadrupole modes.

The present paper gives the results of calculation with MAFIA and measurements of a low power model cavity 
TABLE I. Relation between cutoff frequencies and beam pipe diameter for TESLA cavity.

\begin{tabular}{lccc}
\hline \hline Propagating mode & Dipole TE11 & Monopole TM01 & Quadrupole TE21 \\
\hline Cutoff frequency for $\phi 78$ beam pipe (TESLA design) & $2.25 \mathrm{GHz}$ & $2.94 \mathrm{GHz}$ & $3.74 \mathrm{GHz}$ \\
Lowest HOM frequency of cavity corresponding to propagating mode & $1.62 \mathrm{GHz}$ & $2.34 \mathrm{GHz}$ & $2.23 \mathrm{GHz}$ \\
Minimum beam pipe diameter required for propagation of HOM & $109 \mathrm{~mm}$ & $98 \mathrm{~mm}^{-1}$ & $131 \mathrm{~mm}$ \\
Decay constant for 1.3 GHz fundamental mode with above beam pipe & $35.0 \mathrm{~mm}^{-1}$ & $40.7 \mathrm{~mm}^{-1}$ & $113 \mathrm{~mm}$ \\
Beam pipe length needed for 99\% decay of fundamental mode field & $132 \mathrm{~mm}$ & $186 \mathrm{~mm}$ \\
\hline \hline
\end{tabular}

with the EFB, which confirm the effectiveness of the EFB in damping the quadrupole modes.

\section{ECCENTRIC-FLUTED BEAM PIPE}

\section{A. Principle}

A fluted beam pipe, as shown in Fig. 1(a), can lower the cutoff frequencies of rf modes propagating along the beam pipe. Propagation of quadrupole modes requires a large fluted beam pipe. Furthermore, propagation of the HOMs throughout the beam pipe requires that the flute extend over the entire length of the beam pipe up to the HOM absorber installed at the end of the beam pipe.

An example of the EFB is shown in Fig. 1(b). In this case, the flute is asymmetrically shifted from the center of the beam pipe and the field pattern around the flute can be assumed to include a dipole mode. This suggests that the quadrupole mode can couple with the EFB and propagate as a dipole mode.

To confirm this principle of the EFB, the Fourier components of the rf field along the beam axis were calculated with MAFIA. The model used was a TESLA-type single-cell cavity with eccentric flutes of $60 \mathrm{~mm}$ length, $20 \mathrm{~mm}$ height, $31 \mathrm{~mm}$ width, and $10 \mathrm{~mm}$ shift from the beam pipe center. Figure 2 shows the calculated Fourier compo- (a)

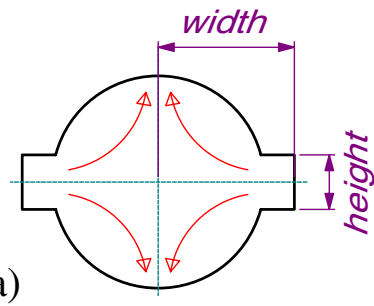

(c)

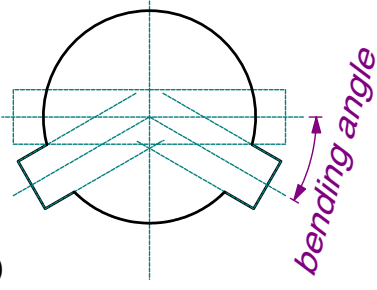

(b)

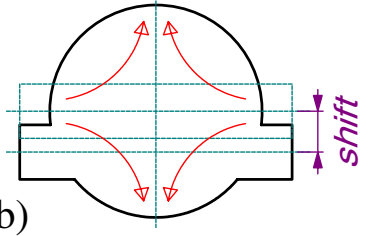

(d)

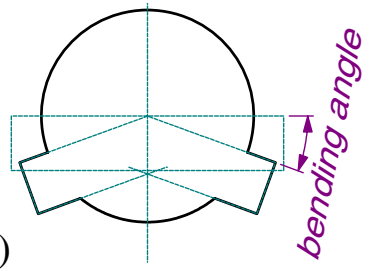

FIG. 1. (Color) Beam pipe cross sections with normal flute (a) and eccentric flute (b)-(d): (b) shifting a flute from the beam pipe center (type 1); (c) bending a flute around the beam pipe center (type 2); (d) combination of shifting and bending a flute (type 3 ). nents of dipole and quadrupole modes when the quadrupole mode is excited in the cavity. The cell was located from 0 to $113 \mathrm{~mm}$ and the eccentric-flute from -14 to $-74 \mathrm{~mm}$. The dipole mode seen here was induced by the eccentric-fluted beam pipe as the rf propagates through this pipe. The fact that the rf field was propagating at the end of the EFB clearly suggests that the EFB acts as a mode converter from quadrupole mode to dipole. It should be noticed that a normal fluted beam pipe cannot convert the field pattern from the quadrupole mode to another mode.

These calculations also revealed that the EFB is effective for both of the degenerate quadrupole modes which rotate 45 degrees around the beam axis, though the effect is not the same for the degenerate quadrupole modes.
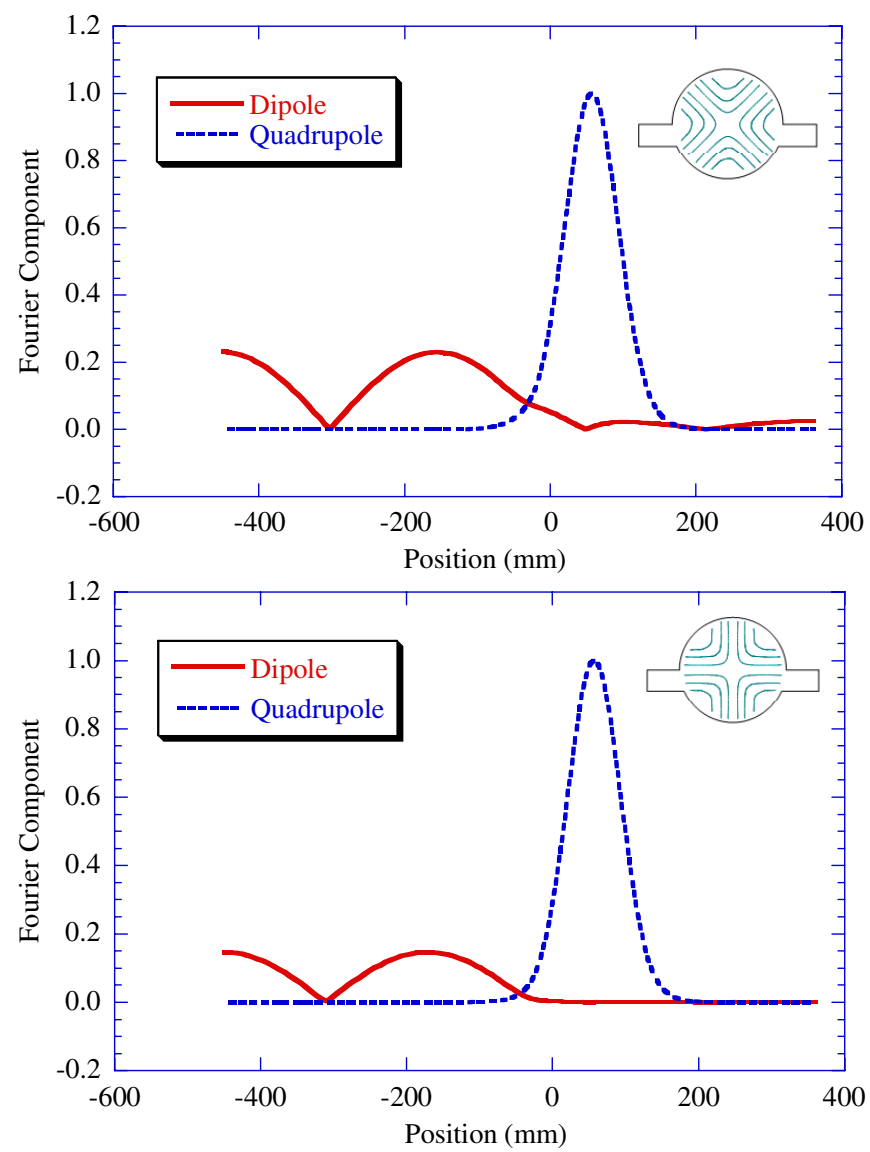

FIG. 2. (Color) Fourier components of electric field of the cross section along the axis. The polarities of quadrupole against the eccentric flute are shown in the figures. 


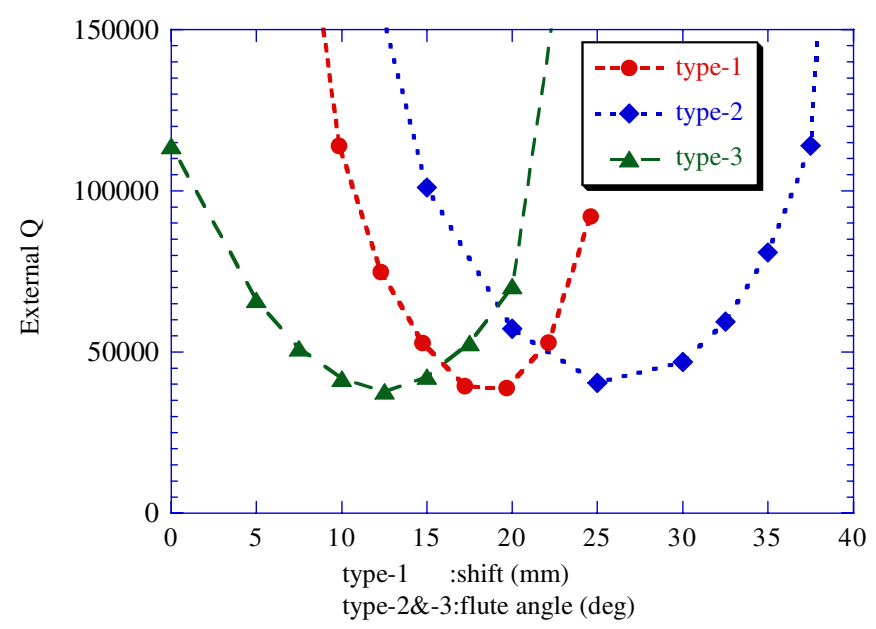

FIG. 3. (Color) Comparison of external $Q$ values of three types of EFPs as a function of shift distance (type 1) and bending angle (types 2 and 3).

\section{B. Method to calculate external $Q$ value}

The properties of the EFB were estimated by calculating external $Q$ values of the EFB. A method to calculate the external $Q$ value with nondissipative codes was proposed elsewhere [8,9]. The external $Q$ value for a line consisting of a TE wave can be calculated by

$$
\begin{gathered}
Q_{\mathrm{ext}}=\frac{2 \pi}{\lambda}\left(\frac{\Lambda}{\lambda} R_{E}+\frac{\lambda}{\Lambda} R_{H}\right), \\
R_{E}=\frac{\iiint|E|^{2} d V}{\iint|E|^{2} d S}, \\
R_{H}=\frac{\iiint|H|^{2} d V}{\iint|H|^{2} d S},
\end{gathered}
$$

where $\lambda$ and $\Lambda$ are, respectively, the wavelengths in a free space and in a waveguide. The volume and surface integrations are performed, respectively, over the cavity volume and over a beam pipe cross section which has an electric field antinode in Eq. (2) and a magnetic field antinode in Eq. (3). These can be calculated with MAFIA.

\section{Eccentric-flute shape}

Three EFB types with different asymmetrical configurations of flutes were considered, as shown in Figs. 1(b)1(d). The first simply shifts the flute from the beam pipe center (type 1), the second bends the flute at the beam pipe center (type 2), and the third is a combination of the above shapes both shifting and bending the flute (type 3 ).

Figure 3 shows the calculated external $Q$ values for the TESLA-type single-cell cavity with the three types of the EFB. The EFB is located $14 \mathrm{~mm}$ from the end of the cell. The EFB is $60 \mathrm{~mm}$ long, $20 \mathrm{~mm}$ high, and $70 \mathrm{~mm}$ wide. The flute for type 3 is shifted by half of the height from the beam pipe center and bent at the beam pipe center. The bending angle of type 3 is defined as plus towards the shifted flute. Since the minimum external $Q$ values for the three types are almost the same, we selected the type 2 EFB because of its ease of fabrication.

To determine the optimum shape of the EFB, the external $Q$ values were calculated for various EFB bending angles, lengths, widths, and positions removed from the cell end.

\section{MEASUREMENT OF $Q_{\text {ext }}$ OF ECCENTRIC FLUTES}

\section{A. Model cavity measurement}

A low power EFB model made of aluminum was fabricated to estimate the external $Q$ value of the EFB. Figure 4 shows the setup for the measurement. The EFB model consisted of seven pieces of angularly divided beam pipe as shown in Fig. 5. The flute angle could be chosen to be from zero to 45 degrees in five-degree increments by changing the order of the pieces. The flute parameters could be chosen as follows: length from 60 to $100 \mathrm{~mm}$ in $10 \mathrm{~mm}$ increments, width from 50 to $85 \mathrm{~mm}$ in $5 \mathrm{~mm}$ increments, and position from 14 to $49 \mathrm{~mm}$ in $5 \mathrm{~mm}$ increments. The EFB was attached to one side of a TESLA three-cell cavity. A drive antenna was installed at the side of one end cell and a pickup antenna was installed at the side of the other end cell at the same angle. Two types of antenna such as a loop and a pin were used to measure the degenerate modes separately. A rolled-up ferrite sheet was set inside the beam pipe as a HOM absorber. Loaded $Q$ values of TE211 modes were measured with a network analyzer with various ferrite positions, defined as the length of the interval indicated in Fig. 4.

\section{B. Method of estimating external $Q$ value}

Figure 6 shows measured examples of loaded $Q$ values of the three-cell cavity with and without the EFB. The flute

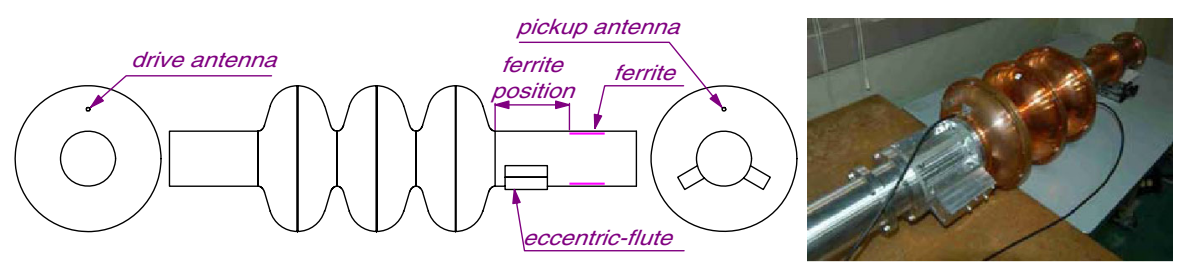

FIG. 4. (Color) Setup for the $Q$ value measurement of the EFB. 


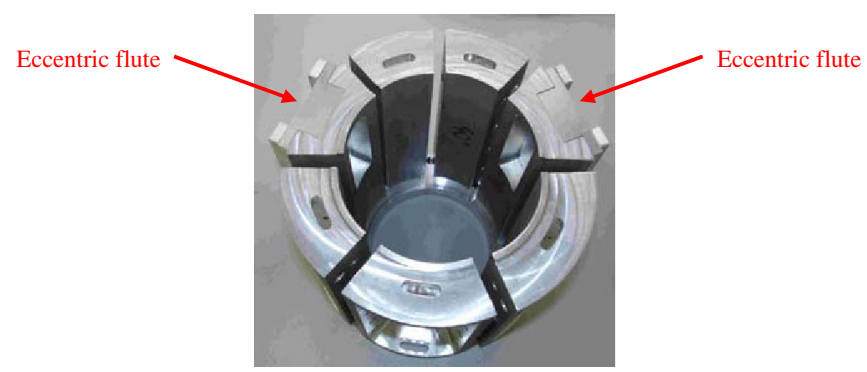

FIG. 5. (Color) Angularly divided eccentric-fluted beam pipe.

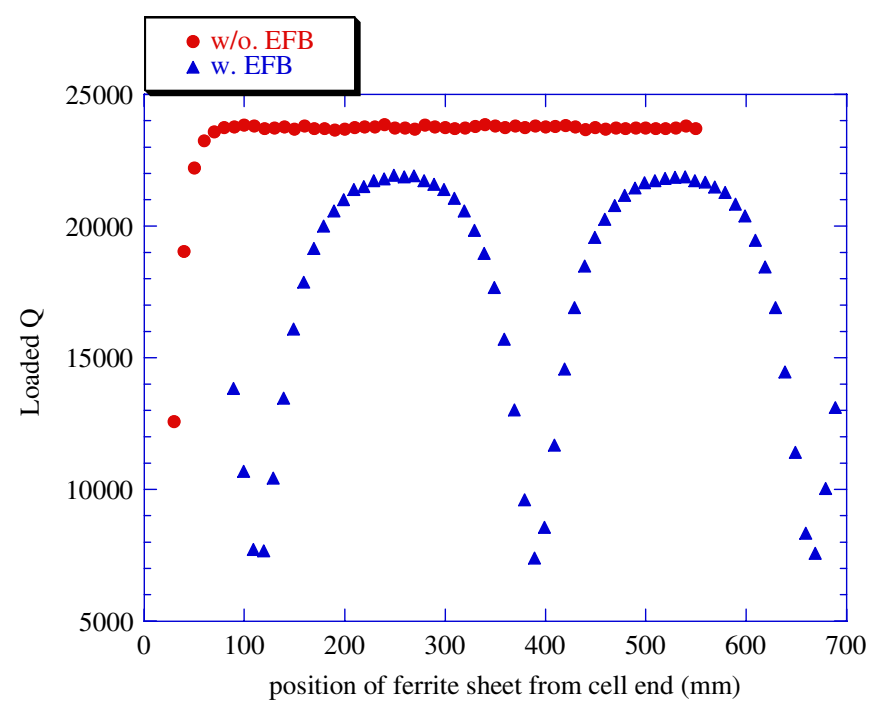

FIG. 6. (Color) The loaded $Q$ values as a function of the ferrite position with and without the EFB.

angle was 25 degrees. The figure shows that the loaded $Q$ value without the EFB remains constant until the ferrite sheet comes close to the cell, at which position the quadrupole mode penetrates into the beam pipe. The loaded $Q$ value with the EFB varied with shift in the ferrite position, even far from the cell. This indicates that in the quadrupole modes, energy propagates through the beam pipe.

If the HOM absorber has ideal properties of perfect absorption and no reflection, the loaded $Q$ values should be constant regardless of the position of the HOM absorber. Since the HOM absorber reflects to some extent in practice,
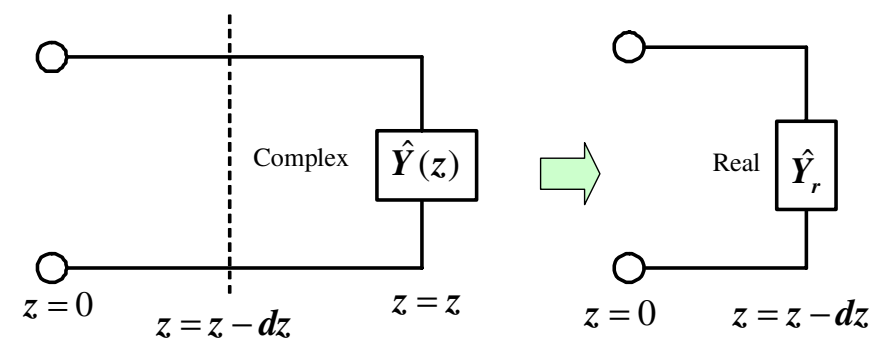

FIG. 7. (Color) Equivalent circuits of beam pipe with the HOM absorber.

however, the loaded $Q$ value varies according to the position of the HOM absorber.

We can estimate the external $Q$ value from the measured loaded $Q$ values by the following procedures. When the load in the transmission line connected with a cavity has a normalized conductance of $g$, unloaded, loaded and external $Q$ values are related by the following equation:

$$
\frac{1}{Q_{L}}=\frac{1}{Q_{0}}+\frac{g}{Q_{\mathrm{ext}}}
$$

where $Q_{L}, Q_{0}$, and $Q_{\text {ext }}$ are loaded, unloaded, and external $Q$ values, respectively. In this expression, it is assumed that external $Q$ values except for the transmission line are added to the value of $Q_{0}$. The normalized conductance $g$ is a function of the position of the HOM absorber.

We assume that the load located at the position $z$ has normalized admittance $\hat{Y}(z)$ as shown in Fig. 7 (left). Although $\hat{Y}(z)$ is complex, we assume that it becomes real viewed at the distance $d z$ from the load. This is equivalent to locating the load of real normalized admittance $\hat{Y}_{r}$ at the position of $z-d z$ as shown in Fig. 7 (right). The normalized admittance of this load viewed from $z=0$ can be expressed as

$$
\hat{Y}(0)=\frac{\hat{Y}_{r}+\tanh \gamma(z-d z)}{1+\hat{Y}_{r} \tanh \gamma(z-d z)},
$$

where $\gamma$ is a propagation constant expressed as $\gamma=\alpha+$ $j \beta, \alpha$ is an attenuation constant, and $\beta$ is a phase constant. The normalized conductance $g$ is the real part of Eq. (5). The normalized conductance can be expressed as

$$
g=\frac{2 \hat{Y}_{r} \cosh 2 \boldsymbol{\alpha}(z-d z)+\left(1+\hat{Y}_{r}^{2}\right) \sinh 2 \boldsymbol{\alpha}(z-d z)}{\left(1+\hat{Y}_{r}^{2}\right) \cosh 2 \boldsymbol{\alpha}(z-d z)+2 \hat{Y}_{r} \sinh 2 \boldsymbol{\alpha}(z-d z)+\left(1-\hat{Y}_{r}^{2}\right) \cos 2 \beta(z-d z)}
$$

The loaded $Q$ value can be expressed as a function of $z$ with the constant parameters of $\hat{Y}_{r}, \alpha, \beta, d z, Q_{0}$, and $Q_{\text {ext }}$ :

$$
\frac{1}{Q_{L}(z)}=\frac{1}{Q_{0}}+\frac{g\left(z ; \hat{Y}_{r}, \boldsymbol{\alpha}, \boldsymbol{\beta}, \boldsymbol{d} z\right)}{Q_{\mathrm{ext}}} .
$$

The method of estimating the external $Q$ value is summarized as follows: (1) Measure the loaded $Q$ values while changing the position of the HOM absorber. (2) Fit the loaded $Q$ values to Eqs. (6) and (7). 
TABLE II. Parameters of the EFB used for the $Q$ value measurement and calculation.

\begin{tabular}{lcccc}
\hline \hline & Figure 8 & Figure 9 & Figure 10 & Figure 11 \\
\hline Angle (deg) & $0-45$ step 5 & 20 & 20 & 20 \\
Width (mm) & 70 & $50-85$ step 5 & 85 & 70 \\
Length (mm) & 60 & 60 & $60-100$ step 10 & 60 \\
Position (mm) & 14 & 14 & 14 & $14-49$ step 5 \\
\hline \hline
\end{tabular}

\section{COMPARISON OF CALCULATIONS AND MEASUREMENTS}

The eccentric-flute parameters which were varied for calculation and measurement are shown in Table II.
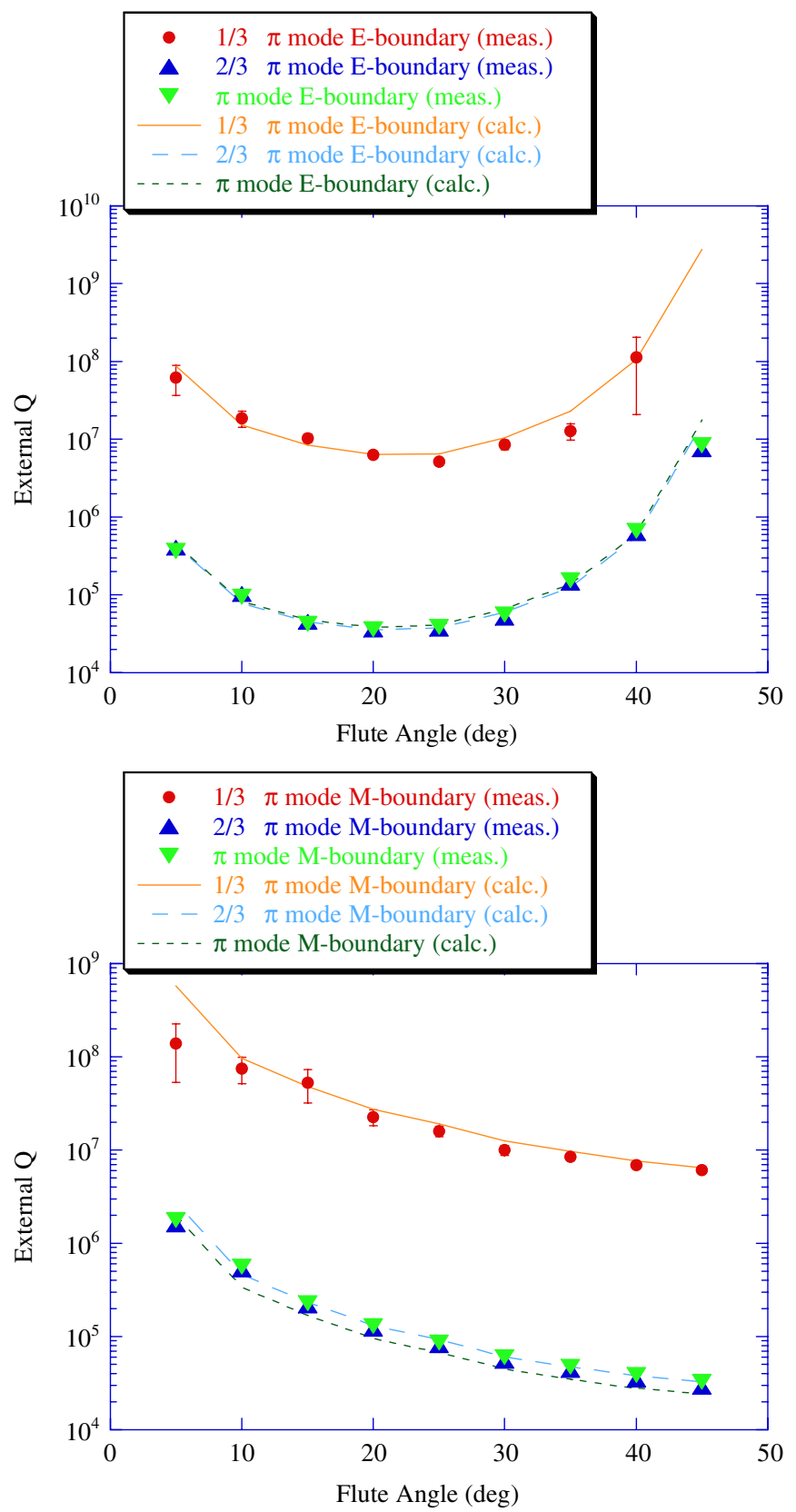

FIG. 8. (Color) Calculated and measured external $Q$ values of three-cell cavity with the EFB as a function of bending angle.
Figures 8-11 show the external $Q$ values of calculation and measurement as a function of flute angle, flute width, flute length, and flute position, respectively.
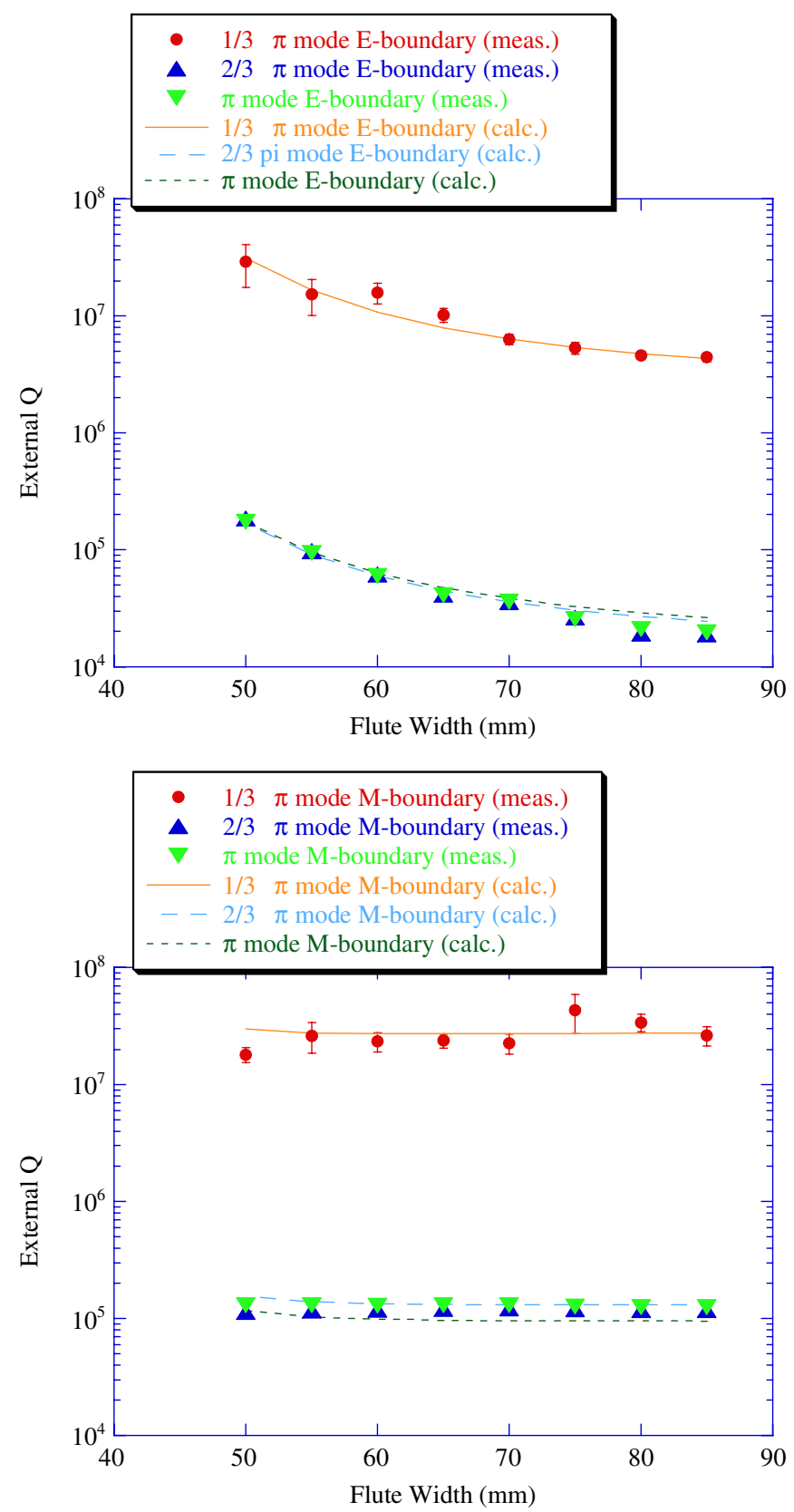

FIG. 9. (Color) Calculated and measured external $Q$ values of three-cell cavity with the EFB as a function of flute width. 

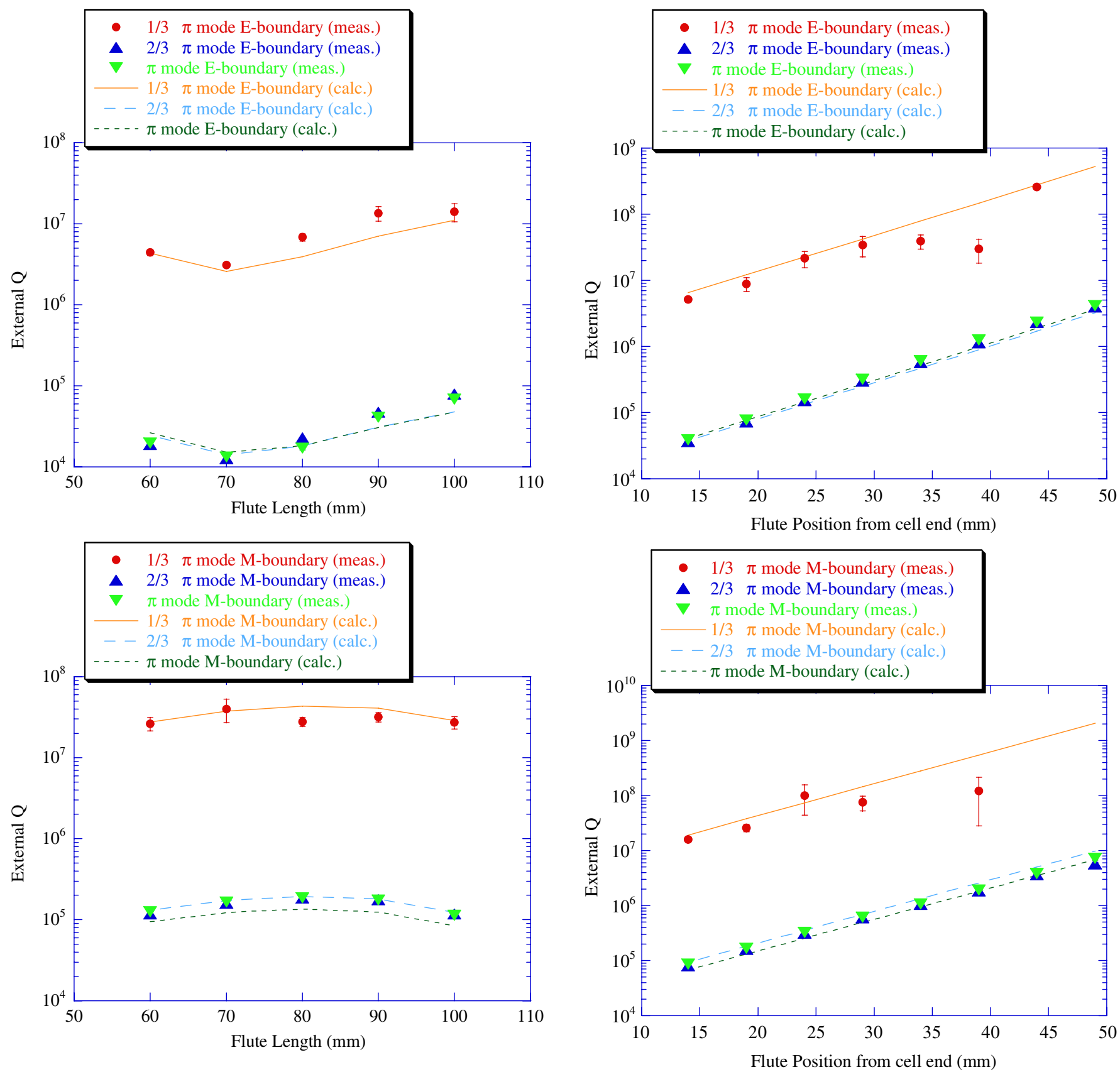

FIG. 10. (Color) Calculated and measured external $Q$ values of three-cell cavity with the EFB as a function of flute length.

The $E$ boundary plotted in the graphs represents the electric boundary conditions of the modes on the plane along the axis symmetrically cutting the EFB, and the $M$ boundary represents the magnetic boundary conditions of the same.

The measurements agreed well with the calculations.

\section{DISCUSSION}

As shown in Figs. 8-11, the external $Q$ values of the $\pi$ and $2 / 3-\pi$ modes were smaller than those of the $1 / 3-\pi$ mode. Since the TESLA-type cavity was designed to en-

FIG. 11. (Color) Calculated and measured external $Q$ values of three-cell cavity with the EFB as a function of flute position from the cell end.

hance some HOMs in one end cell and others in the other end cell through asymmetric end cell shaping [6], the field strength of all cells was not equal. In our setup, the TE211 $1 / 3-\pi$ mode excited a weaker cell field at the side of the EFB near the cells than at the far side, as shown in Fig. 12. The weak cell field at the near side of the EFB caused weak coupling between the field and the EFB. This resulted in an external $Q$ value of the TE211 1/3- $\pi$ mode larger than those of the $\pi$ and 2/3- $\pi$ modes. When the field distribution is not uniform due to the asymmetric shape of the end 

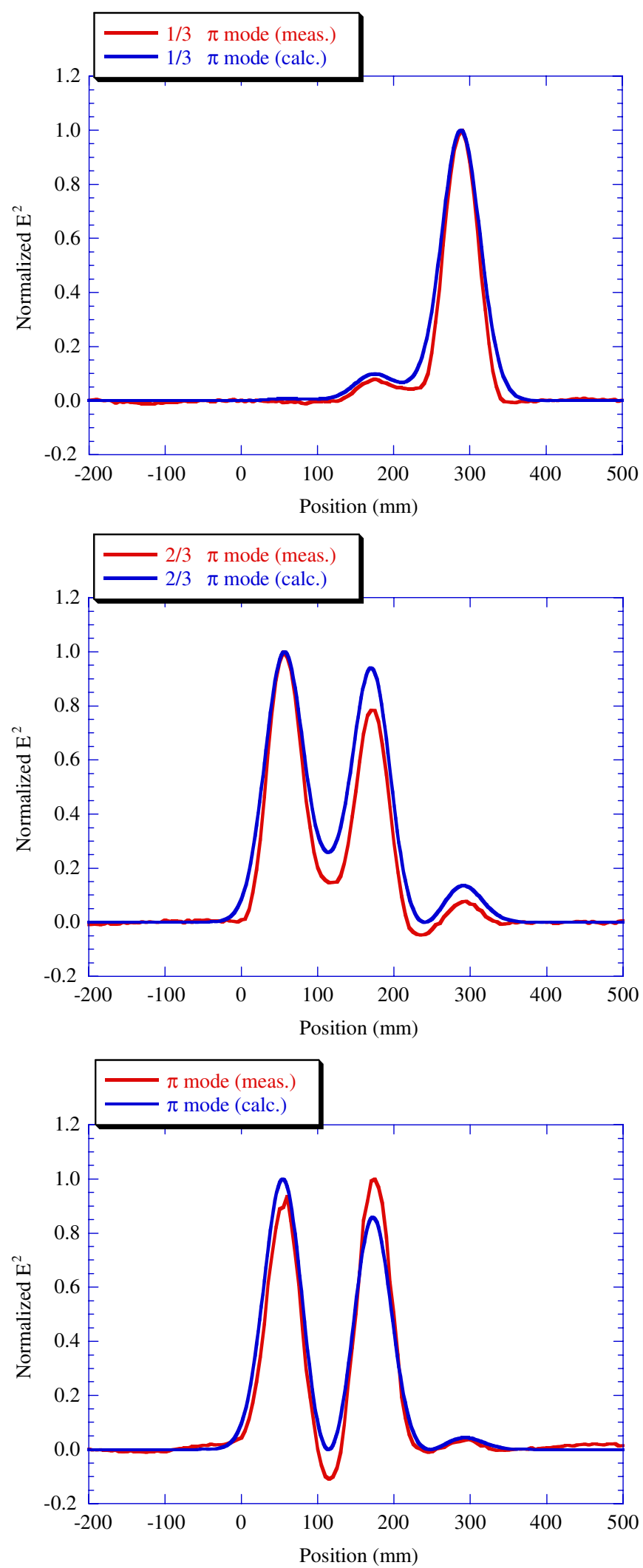

FIG. 12. (Color) Calculated and measured field distributions of TE211 $1 / 3$ pi (top), $2 / 3$ pi (middle), and pi (bottom). The eccentric flute is located from -14 to $-114 \mathrm{~mm}$. The cells are located from 0 to $344 \mathrm{~mm}$.

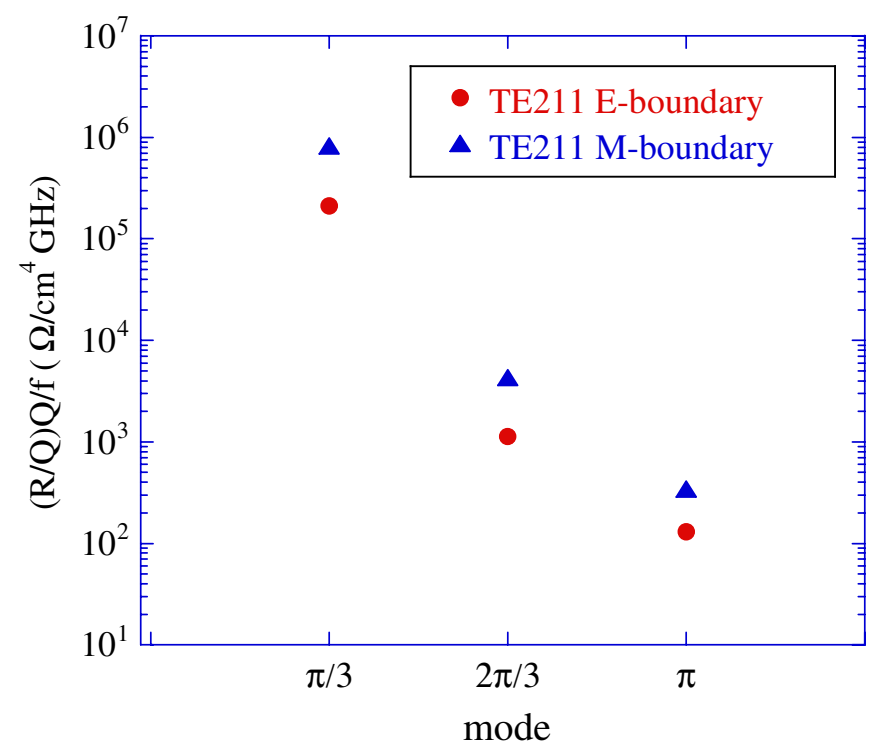

FIG. 13. (Color) The calculated values of $(R / Q) Q / f$ for TE211 modes of TESLA-type three-cell cavity with the EFB.

cell, and the quadrupole field at the end cell on the EFB side is small, installing EFBs into both ends of the beam pipes enables damping of all the quadrupole modes.

The quadrupole mode criterion for the $100 \mathrm{~mA}$ ERL designed by the Cornell University group [5] was $(R / Q) Q / f<4 \times 10^{6} \Omega / \mathrm{cm}^{4} \mathrm{GHz}$, where $R, Q$, and $f$ are shunt impedance, $Q$ value and frequency, respectively. The calculated values of $(R / Q) Q / f$ for the TE211 modes of a TESLA-type three-cell cavity with the EFB meet this criterion as shown in Fig. 13. The flute is $60 \mathrm{~mm}$ long, $20 \mathrm{~mm}$ high, $70 \mathrm{~mm}$ wide, 20 degrees angular, and located at $14 \mathrm{~mm}$ from the end of the cell. Since the seven-cell or nine-cell cavity is used for a real machine, the values of $(R / Q) Q / f$ were calculated for the TESLA-type nine-cell cavity with the EFB of the same parameters as shown in Fig. 14. Though the values of $(R / Q) Q / f$ were worse for the nine-cell cavity than those for the three-cell cavity, all modes except for the TE211 $1 / 9-\pi$ mode meet the criterion. Similar to the three-cell cavity, the TE211 1/9- $\pi$ mode of the TESLA-type nine-cell cavity weakly couples with the EFB due to asymmetry end cell shaping. Installing the EFBs into both ends of beam pipes and/or improving the field flatness of HOMs will reduce values of $(R / Q) Q / f$ for the HOMs including the $1 / 9-\pi$ mode and realize a cavity design to meet the criterion for a $100 \mathrm{~mA}$ ERL.

The flute length dependence shown in Fig. 10 indicates that a long EFB is not always effective in damping the quadrupole, since a long EFB can increase the external $Q$ value. Since the EFB works as the mode converter from the quadrupole to the dipole, it can be also considered to be a mode converter from the dipole mode to quadrupole. This means that a long EFB may reconvert the dipole mode to the quadrupole mode. Calculated external $Q$ values with the longer EFB are shown in Fig. 15. The external $Q$ values 


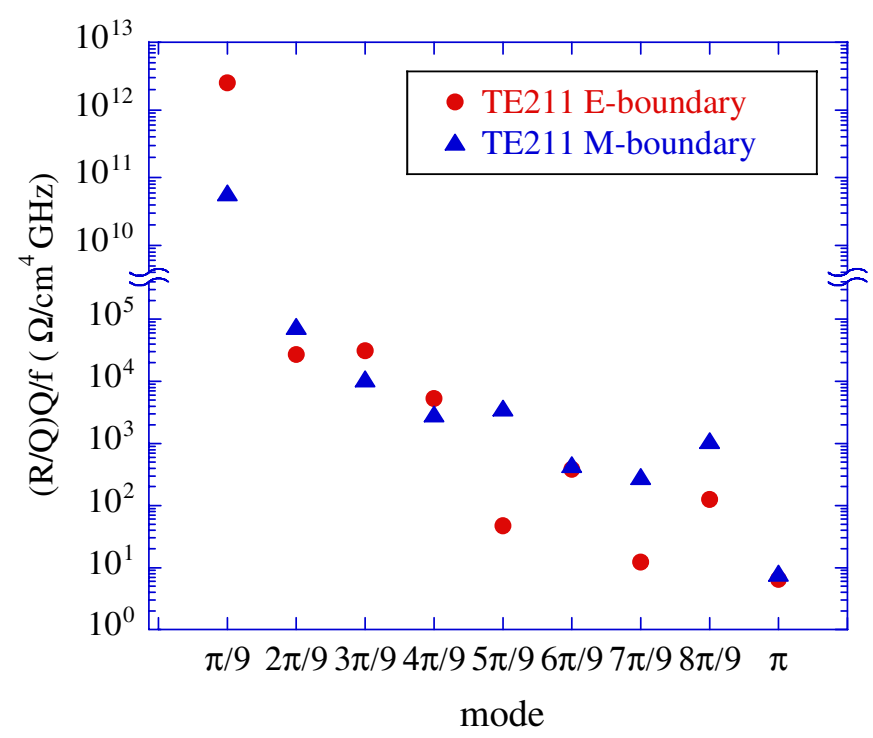

FIG. 14. (Color) The calculated values of $(R / Q) Q / f$ for TE211 modes of TESLA-type nine-cell cavity with the EFB.

varied periodically according to the EFB length. Figure 16 shows the calculated Fourier components along the beam axis with the EFB of $230 \mathrm{~mm}$ length, 30 degrees angle, and $70 \mathrm{~mm}$ width, and located from -14 to $-244 \mathrm{~mm}$. These indicate that the quadrupole mode decreases at the entrance of the beam pipe because there is a lower frequency than the cutoff frequency, and that the quadrupole mode increases as the position gets farther from where there is quadrupole attenuation. The quadrupole at such a removed position is considered to be reconverted from the dipole mode.

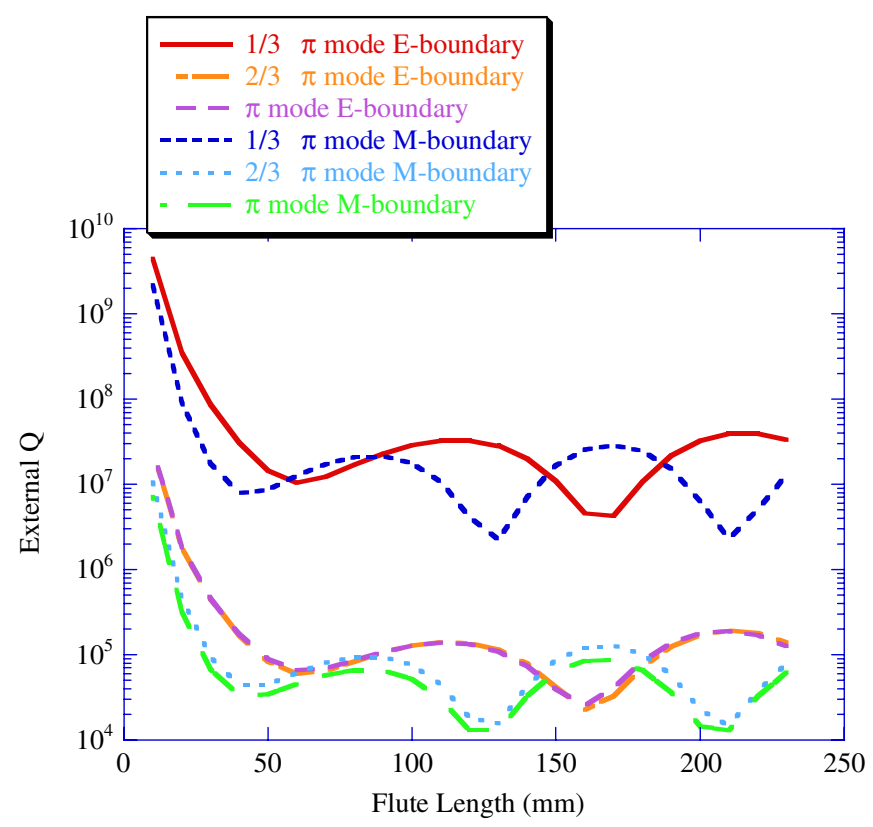

FIG. 15. (Color) Calculated external $Q$ values of the long EFB as a function of flute length.

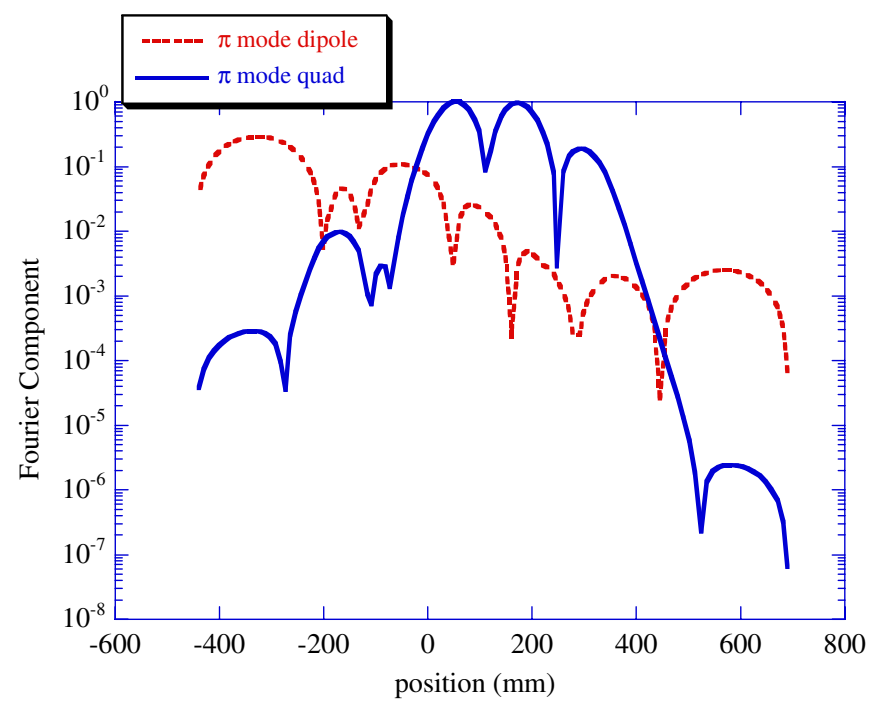

FIG. 16. (Color) Calculated Fourier components of electric field of the cross section along the axis for the long EFB.

\section{CONCLUSION}

Much investigation has been made into a superconducting cavity which does not have multipass BBU instabilities due to HOMs. A HOM free cavity for the electron cooling project with ERL with an optimized cavity shape and beam pipes with ferrite absorbers has been proposed [10]. Although that cavity can damp the dipole and the monopole modes sufficiently, the quadrupole modes are not damped. The EFB we developed is effective in damping the quadrupole modes.

Since quadrupole modes are converted into dipole modes by EFB and penetrate from the cavity into the beam pipe, the optimum structure of the EFB is virtually uninfluenced by the cavity shape design. The EFB is in principle independent of the number of cells of the cavity, so that the design of the EFB can easily be adapted to the typical nine-cell cavity. A suitable EFB can be installed at any type of cavity optimized for dipole damping as far as quadrupole modes penetrate into a beam pipe. After installation of the EFB, the quadruple modes will be damped by the absorber at the beam pipe together with the dipole modes.

The EFB makes it possible to damp the quadrupole modes which cannot be effectively damped by enlarging the beam pipes and optimizing cell shapes, and thus to increase the threshold current which is limited by quadrupole BBU instabilities. This realizes a high-current ERL machine of over $100 \mathrm{~mA}$, something not possible heretofore due to quadrupole modes trapped in a cavity.

The ERL project in Japan is proceeding with the cooperation of JAEA, KEK, ISSP, and other SR institutes to realize $5 \mathrm{GeV}$ class ERLs [11-14]. The superconducting main linac with a nine-cell cavity has been designed to suppress the monopole and dipole HOM BBU instabilities 
by optimizing the cell shapes with enlarged beam pipes [15-17]. A BBU simulation showed that this cavity can strongly damp the dipole modes and thus achieve over $600 \mathrm{~mA}$ beam current without HOM randomization [18]. Installing the EFB in this cavity is expected to satisfy the requirement of our ERL machine for strong damping of monopole, dipole, and quadrupole modes. A niobium test cavity with the EFB based on our design has been fabricated, and the measurement of cavity performance is under way.

[1] T. Furuya, Rev. Accel. Sci. Technol. 1, 211 (2008).

[2] E. Pozdeyev, C. Tennant, J. J. Bisognano, M. Sawamura, R. Hajima, and T. I. Smith, Nucl. Instrum. Methods Phys. Res., Sect. A 557, 176 (2006).

[3] L. Merminga, I. E. Campisi, D. R. Douglas, G. A. Krafft, J. Preble, and B.C. Yunn, in Proceedings of the Particle Accelerator Conference, Chicago, IL, 2001 (IEEE, New York, 2001), pp. 173-175.

[4] G. H. Hoffstaetter and I. V. Bazarov, Phys. Rev. ST Accel. Beams 7, 054401 (2004).

[5] M. Liepe, in Proceedings of the 11th Workshop on Superconductivity (SRF2003), Lubeck/Travemunde, 2003, paper MoP33, http://srf2003.desy.de/.

[6] B. Aune et al., Phys. Rev. ST Accel. Beams 3, 092001 (2000).

[7] R. Rimmer, H. Wang, and G. Wu, in Proceedings of the 11th Workshop on Superconductivity (SRF2003), Lubeck/ Travemunde, 2003, paper TuP36, http://srf2003.desy.de/.

[8] P. Balleyguier, in Proceedings of XIX International Linear Accelerator Conference, Chicago, 1998, pp. 133-135.

[9] V. Shemelin and S. Belomestnykh, Calculation of the BCell Cavity External $Q$ with MAFIA and MICROWAVE STUDIO, Cornell Publication No. SRF020620-03.

[10] R. Calaga, I. Ben-Zvi, Y. Zhao, D. Wang, J. Sekutowicz, and G. Wu, in Proceedings of the 11th workshop on
Superconductivity (SRF2003), Lubeck/Travemunde, 2003, paper TuP05, http://srf2003.desy.de/.

[11] T. Kasuga et al., in Proceedings of the 2007 Particle Accelerator Conference, Albuquerque, New Mexico, 2007 (IEEE, Albuquerque, New Mexico, 2007), pp. 1016-1018, paper TUPMN044, http://www. JACoW.org/.

[12] T. Kasuga et al., in Proceedings of the 4th Asian Particle Accelerator Conference, Indore, 2007 (RRCAT, Indore, India, 2007), pp. 172-174, paper TUPMA046, http:// www.JACoW.org/.

[13] S. Sakanaka et al., in Proceedings of the 11th European Particle Accelerator Conference, Genoa, 2008 (EPS-AG, Genoa, Italy, 2008), pp. 205-207, paper MOPC061, http:// www.JACoW.org/.

[14] R. Hajima, N. Nakamura, S. Sakanaka, and Y. Kobayashi, KEK Report No. 2007-7, JAEA-Research 2008-032, 2008 (in Japanese).

[15] H. Sakai, K. Shinoe, T. Furuya, S. Sakanaka, T. Suwada, T. Takahashi, K. Umemori, and M. Sawamura, in Proceedings of 41st Advanced ICFA Beam Dynamics Workshop on Energy Recovery Linacs, Daresbury, 2007, pp. 56-61, http://www.JACoW.org/.

[16] K. Umemori, T. Furuya, T. Takahashi, H. Sakai, K. Shinoe, and M. Sawamura, in Proceedings of the 11th European Particle Accelerator Conference, Genoa, 2008 (Ref. [13]), pp. 925-927, paper MOPP159, http:// www.JACoW.org/.

[17] H. Sakai, K. Shinoe, T. Furuya, T. Takahashi, K. Umemori, and M. Sawamura, in Proceedings of the 11th European Particle Accelerator Conference, Genoa, 2008 (Ref. [13]), pp. 907-909, paper MOPP153, http:// www.JACoW.org/.

[18] R. Hajima and R. Nagai, Analysis of HOM-BBU with Newly Designed Cavities for a Multi-GeV ERL Light Source, in Proceedings of 41st Advanced ICFA Beam Dynamics Workshop on Energy Recovery Linacs, Daresbury, 2007, pp. 133-138, http://www.JACoW.org/. 